\title{
Global Stability and Hopf Bifurcation of a Predator-Prey Model with Time Delay and Stage Structure
}

\author{
Lingshu Wang ${ }^{1}$ and Guanghui Feng ${ }^{2}$ \\ ${ }^{1}$ School of Mathematics and Statistics, Hebei University of Economics \& Business, Shijiazhuang 050061, China \\ ${ }^{2}$ Department of Basic Courses, Shijiazhuang Mechanical Engineering College, Shijiazhuang 050003, China \\ Correspondence should be addressed to Lingshu Wang; wanglingshu@126.com
}

Received 10 June 2013; Accepted 6 January 2014; Published 25 February 2014

Academic Editor: Keshlan S. Govinder

Copyright (c) 2014 L. Wang and G. Feng. This is an open access article distributed under the Creative Commons Attribution License, which permits unrestricted use, distribution, and reproduction in any medium, provided the original work is properly cited.

\begin{abstract}
A delayed predator-prey system with Holling type II functional response and stage structure for both the predator and the prey is investigated. By analyzing the corresponding characteristic equations, the local stability of each of the feasible equilibria of the system is addressed and the existence of a Hopf bifurcation at the coexistence equilibrium is established. By means of persistence theory on infinite dimensional systems, it is proved that the system is permanent. By using Lyapunov functions and the LaSalle invariant principle, the global stability of each of the feasible equilibria of the model is discussed. Numerical simulations are carried out to illustrate the main theoretical results.
\end{abstract}

\section{Introduction}

The predator-prey system is very important in population modelling and has been studied by many authors (see, e.g., [1-6]). A predator-prey model generally takes the form

$$
\begin{gathered}
\dot{x}=x f(x)-p(x) y, \\
\dot{y}=k p(x) y-y g(y),
\end{gathered}
$$

where $x(t)$ and $y(t)$ are the densities of prey and predator populations at time $t$, respectively. The function $f(x)$ represents the growth rate of the prey; $g(y)$ represents the death rate and intraspecific competition rate of the predator; $p(x)$ denotes the predator response function. In 1965, Holling [7] used the function $p(x)=m x /(a+x)$ as one of the predator response functions. It is now referred to as a Holling type II functional response. We note that in the models mentioned above, it is assumed that both the immature and the mature predators have the same ability to attack prey individuals. However, in the real world, almost all animals have stage structure of immature and mature, and only mature predators can attack prey and have reproductive ability. Stage-structured models have received great attention in recent years (see, e.g., [2-6]). In [2], Wang proposed a predator-prey system with Holling type II functional response and stage structure under the assumptions that the predator is divided into two groups, one is immature and the other is mature, and that only mature predators can attack prey and have reproductive ability, while immature predators do not attack prey and have no reproductive ability.

It is generally recognized that some kinds of time delays are inevitable in population interactions and tend to be destabilizing in the sense that longer delays may destroy the stability of positive equilibria (see [8]). Time delay due to gestation is a common example, because generally the consumption of prey by the predator throughout its past history governs the present birth rate of the predator. Recently, great attention has been received and a large body of work has been carried out on the existence of Hopf bifurcations in delayed population models (see, e.g., $[5,6,8,9]$ and references cited therein).

Motivated by the work of $[2,6]$, in the present paper, we are concerned with the combined effects of stage structure for both the predator and the prey and time delay due to the gestation of the predator on the global dynamics of a predator-prey model with Holling type II functional 
response. To this end, we consider the following differential system:

$$
\begin{gathered}
\dot{x}_{1}(t)=r x_{2}(t)-\left(r_{1}+d_{1}\right) x_{1}(t)-a x_{1}^{2}(t)-\frac{a_{1} x_{1}(t) y_{2}(t)}{1+m x_{1}(t)}, \\
\dot{x}_{2}(t)=r_{1} x_{1}(t)-d_{2} x_{2}(t), \\
\dot{y}_{1}(t)=\frac{a_{2} x_{1}(t-\tau) y_{2}(t-\tau)}{1+m x_{1}(t-\tau)}-\left(r_{2}+d_{3}\right) y_{1}(t), \\
\dot{y}_{2}(t)=r_{2} y_{1}(t)-d_{4} y_{2}(t),
\end{gathered}
$$

where $x_{1}(t)$ and $x_{2}(t)$ represent the densities of the immature and the mature prey at time $t$, respectively; $y_{1}(t)$ and $y_{2}(t)$ represent the densities of the immature and the mature predators at time $t$, respectively. The parameters $a, a_{1}, a_{2}, d_{1}$, $d_{2}, d_{3}, d_{4}, r, r_{1}$, and $r_{2}$ are positive constants, in which $r$ is the birth rate of the prey; $a$ is the intraspecific competition rate of the mature prey; $d_{1}, d_{2}, d_{3}$, and $d_{4}$ are the death rates of the immature prey, mature prey, immature predators, and mature predators, respectively; $r_{1}$ and $r_{2}$ are the transformation rates from the immature individuals to mature individuals for the prey and the predators, respectively; $a_{1}$ is the capturing rate of the predators; $a_{2} / a_{1}$ is the conversion rate of nutrients into the reproduction of the predators; $\tau \geq 0$ is a constant delay due to the gestation of the predators. It is assumed in (2) that the mature individual predators feed on immature prey and have the ability to reproduce.

The initial conditions for system (2) take the form

$$
\begin{array}{cc}
x_{1}(\theta)=\phi_{1}(\theta) \geq 0, & x_{2}(\theta)=\phi_{2}(\theta) \geq 0, \\
y_{1}(\theta)=\varphi_{1}(\theta) \geq 0, & y_{2}(\theta)=\varphi_{2}(\theta) \geq 0, \\
\theta \in[-\tau, 0), \\
\phi_{1}(0)>0, \quad \phi_{2}(0)>0, & \varphi_{1}(0)>0, \quad \varphi_{2}(0)>0, \\
\left(\phi_{1}(\theta), \phi_{2}(\theta), \varphi_{1}(\theta), \varphi_{2}(\theta)\right) \in C\left([-\tau, 0], R_{+0}^{4}\right),
\end{array}
$$

where $R_{+0}^{4}=\left\{\left(x_{1}, x_{2}, x_{3}, x_{4}\right): x_{i} \geq 0, i=1,2,3,4\right\}$.

It is well known by the fundamental theory of functional differential equations [10] that system (2) has a unique solution $\left(x_{1}(t), x_{2}(t), y_{1}(t), y_{2}(t)\right)$ satisfying initial conditions (3). It is easy to show that all solutions of system (2) corresponding to initial conditions (3) are defined on $[0,+\infty]$ and remain positive for all $t \geq 0$.

The organization of this paper is as follows. In the next section, we investigate the local stability of each of the feasible equilibria of system (2). The existence of a Hopf bifurcation at the coexistence equilibrium is studied. In Section 3, by means of persistence theory on infinite dimensional systems, we prove that system (2) is permanent when the coexistence equilibrium exists. In Section 4, by using Lyapunov functionals and the LaSalle invariant principle, we show that both the prey and the predators go to extinction, if both the predatorextinction equilibrium and the coexistence equilibrium are not feasible, and that the predator-extinction equilibrium is globally asymptotically stable when the coexistence equilibrium does not exist, and sufficient conditions are obtained for the global asymptotic stability of the coexistence equilibrium of system (2). A brief discussion is given in Section 5 to conclude this work.

\section{Local Stability}

In this section, we discuss the local stability of each equilibrium of system (2) and the existence of a Hopf bifurcation. It is easy to show that system (2) always has a trivial equilibrium $E_{0}(0,0,0,0)$ and a predator-extinction equilibrium $E_{1}\left(x_{1}^{+}, x_{2}^{+}, 0,0\right)$ when $r r_{1}>d_{2}\left(r_{1}+d_{1}\right)$, where

$$
x_{1}^{+}=\frac{r r_{1}-d_{2}\left(r_{1}+d_{1}\right)}{a d_{2}}, \quad x_{2}^{+}=\frac{r_{1}\left[r r_{1}-d_{2}\left(r_{1}+d_{1}\right)\right]}{a d_{2}^{2}} .
$$

Furthermore, if the following holds:

$$
\begin{aligned}
& \left(H_{1}\right) \quad\left(r r_{1}-d_{2}\left(r_{1}+d_{1}\right)\right) / a d_{2}>d_{4}\left(r_{2}+d_{3}\right) /\left(a_{2} r_{2}-m d_{4}\left(r_{2}+\right.\right. \\
& \left.\left.d_{3}\right)\right)>0,
\end{aligned}
$$

then system (2) has a unique coexistence equilibrium $E^{*}\left(x_{1}^{*}\right.$, $\left.x_{2}^{*}, y_{1}^{*}, y_{2}^{*}\right)$, where

$$
\begin{gathered}
x_{1}^{*}=\frac{d_{4}\left(r_{2}+d_{3}\right)}{a_{2} r_{2}-m d_{4}\left(r_{2}+d_{3}\right)}, \quad x_{2}^{*}=\frac{r_{1}}{d_{2}} x_{1}^{*}, \\
y_{1}^{*}=\frac{d_{4}}{r_{2}} y_{2}^{*}, \\
y_{2}^{*}=\frac{\left(1+m x_{1}^{*}\right)\left[r r_{1}-d_{2}\left(r_{1}+d_{1}\right)-a d_{2} x_{1}^{*}\right]}{a_{1} d_{2}} .
\end{gathered}
$$

The characteristic equation of system (2) at the equilibrium $E_{0}(0,0,0,0)$ is of the form

$$
\begin{aligned}
& {\left[\lambda^{2}+\left(r_{1}+d_{1}+d_{2}\right) \lambda+d_{2}\left(r_{1}+d_{1}\right)-r r_{1}\right]} \\
& \quad \times\left[\lambda^{2}+\left(r_{2}+d_{3}+d_{4}\right) \lambda+d_{4}\left(r_{2}+d_{3}\right)\right]=0 .
\end{aligned}
$$

It is readily seen from (6) that if $r r_{1}<d_{2}\left(r_{1}+d_{2}\right)$, then $E_{0}$ is locally asymptotically stable; if $r r_{1}>d_{2}\left(r_{1}+d_{2}\right)$, then $E_{0}$ is unstable.

The characteristic equation of system (2) at the equilibrium $E_{1}\left(x_{1}^{+}, x_{2}^{+}, 0,0\right)$ takes the form

$$
\begin{gathered}
{\left[\lambda^{2}+\left(r_{1}+d_{1}+d_{2}+2 a x_{1}^{+}\right) \lambda+r r_{1}-d_{2}\left(r_{1}+d_{1}\right)\right]} \\
\times\left[\lambda^{2}+p_{1} \lambda+p_{0}+q_{0} e^{-\lambda \tau}\right]=0 \\
p_{1}=r_{2}+d_{3}+d_{4}, \quad p_{0}=d_{4}\left(r_{2}+d_{3}\right), \\
q_{0}=-\frac{a_{2} r_{2} x_{1}^{+}}{1+m x_{1}^{+}} .
\end{gathered}
$$

It is easy to show that roots of $\lambda^{2}+\left(r_{1}+d_{1}+d_{2}+2 a x_{1}^{+}\right) \lambda+r r_{1}-$ $d_{2}\left(r_{1}+d_{1}\right)=0$ have only negative real parts if $r r_{1}>d_{2}\left(r_{1}+d_{2}\right)$. If $\left(H_{1}\right)$ holds, we have $p_{0}+q_{0}<0$; thus (7) has at least one 
positive real root. Therefore, $E_{1}$ is unstable. If $0<\left(r r_{1}-d_{2}\left(r_{1}+\right.\right.$ $\left.\left.d_{1}\right)\right) /\left(a d_{2}\right)<d_{4}\left(r_{2}+d_{3}\right) /\left(a_{2} r_{2}-m d_{4}\left(r_{2}+d_{3}\right)\right)$, we have $p_{0}+$ $q_{0}>0$; then the equilibrium $E_{1}$ is locally asymptotically stable when $\tau=0$. It is easy to show that $p_{1}^{2}-2 p_{0}>0, p_{0}^{2}-q_{0}^{2}>0$. Therefore, if $0<\left(r r_{1}-d_{2}\left(r_{1}+d_{1}\right)\right) /\left(a d_{2}\right)<d_{4}\left(r_{2}+d_{3}\right) /\left(a_{2} r_{2}-\right.$ $\left.m d_{4}\left(r_{2}+d_{3}\right)\right)$, by Lemma B in Kuang and So [1], we see that the equilibrium $E_{1}$ is locally asymptotically stable for all $\tau>0$.

The characteristic equation of system (2) at the equilibrium $E^{*}$ is of the form

$$
\lambda^{4}+p_{3} \lambda^{3}+p_{2} \lambda^{2}+p_{1} \lambda+p_{0}+\left(q_{2} \lambda^{2}+q_{1} \lambda+q_{0}\right) e^{-\lambda \tau}=0,
$$

where

$$
\begin{gathered}
p_{3}=\alpha+d_{2}+r_{2}+d_{3}+d_{4}, \\
p_{2}=\left(\alpha+d_{2}\right)\left(r_{2}+d_{3}+d_{4}\right)+d_{4}\left(r_{2}+d_{3}\right)+\alpha d_{2}-r r_{1}, \\
p_{1}=\left(r_{2}+d_{3}+d_{4}\right)\left(\alpha d_{2}-r r_{1}\right)+d_{4}\left(r_{2}+d_{3}\right)\left(\alpha+d_{2}\right), \\
p_{0}=d_{4}\left(r_{2}+d_{3}\right)\left(\alpha d_{2}-r r_{1}\right), \quad q_{2}=-d_{4}\left(r_{2}+d_{3}\right), \\
q_{1}=d_{4}\left(r_{2}+d_{3}\right)\left[\frac{a_{1} y_{2}^{*}}{\left(1+m x_{1}^{*}\right)^{2}}-\left(\alpha+d_{2}\right)\right], \\
q_{0}=d_{4}\left(r_{2}+d_{3}\right)\left[\frac{a_{1} d_{2} y_{2}^{*}}{\left(1+m x_{1}^{*}\right)^{2}}+r r_{1}-\alpha d_{2}\right], \\
\alpha=r_{1}+d_{1}+2 a x_{1}^{*}+\frac{a_{1} y_{2}^{*}}{\left(1+m x_{1}^{*}\right)^{2}} .
\end{gathered}
$$

When $\tau=0$, (8) becomes

$$
\lambda^{4}+p_{3} \lambda^{3}+\left(p_{2}+q_{2}\right) \lambda^{2}+\left(p_{1}+q_{1}\right) \lambda+p_{0}+q_{0}=0 .
$$

By calculation we derive that

$$
p_{3}>0, \quad p_{0}+q_{0}=d_{4}\left(r_{2}+d_{3}\right) \frac{a_{1} d_{2} y_{2}^{*}}{\left(1+m x_{1}^{*}\right)^{2}}>0 .
$$

Hence, by the Routh-Hurwitz criterion, we see that if the following hold:

$$
\begin{aligned}
\left(H_{2}\right) & p_{3}\left(p_{2}+q_{2}\right)>\left(p_{1}+q_{1}\right), p_{3}\left(p_{2}+q_{2}\right)\left(p_{1}+q_{1}\right)> \\
& \left(p_{1}+q_{1}\right)^{2}+p_{3}^{2}\left(p_{0}+q_{0}\right),
\end{aligned}
$$

then the equilibrium $E^{*}$ is locally asymptotically stable when $\tau=0$.

If $i \omega(\omega>0)$ is a solution of (8), separating real and imaginary parts, we have

$$
\begin{gathered}
\left(q_{2} \omega^{2}-q_{0}\right) \cos \omega \tau-q_{1} \omega \sin \omega \tau=\omega^{4}-p_{2} \omega^{2}+p_{0}, \\
\left(q_{2} \omega^{2}-q_{0}\right) \sin \omega \tau+q_{1} \omega \cos \omega \tau=p_{3} \omega^{3}-p_{1} \omega .
\end{gathered}
$$

Squaring and adding the two equations of (12), it follows that

$$
\omega^{8}+h_{3} \omega^{6}+h_{2} \omega^{4}+h_{1} \omega^{2}+h_{0}=0 .
$$

It is easy to show that

$$
\begin{aligned}
h_{3}= & p_{3}^{2}-2 p_{2}=\alpha^{2}+d_{2}^{2}+\left(r_{2}+d_{3}\right)^{2}+d_{4}^{2}+2 r r_{1}>0, \\
h_{2}= & p_{2}^{2}+2 p_{0}-2 p_{1} p_{3}-q_{2}^{2} \\
= & \left(\alpha d_{2}-r r_{1}\right)^{2}+\left[d_{4}^{2}+\left(r_{2}+d_{3}\right)^{2}\right]\left(\alpha^{2}+d_{2}^{2}+2 r r_{1}\right) \\
> & 0, \\
h_{1}= & p_{1}^{2}-2 p_{0} p_{2}+2 q_{0} q_{2}-q_{1}^{2} \\
= & \left(\alpha d_{2}-r r_{1}\right)^{2}\left[d_{4}^{2}+\left(r_{2}+d_{3}\right)^{2}\right]+d_{4}^{2}\left(r_{2}+d_{3}\right)^{2} \\
& \times \frac{a_{1} y_{2}^{*}}{\left(1+m x_{1}^{*}\right)^{2}}\left[2\left(r_{1}+d_{1}\right)+4 a x_{1}^{*}+\frac{a_{1} y_{2}^{*}}{\left(1+m x_{1}^{*}\right)^{2}}\right] \\
> & 0, \quad \\
\quad h_{0}= & p_{0}^{2}-q_{0}^{2}=d_{4}^{2}\left(r_{2}+d_{3}\right)^{2} \frac{a_{1} d_{2} y_{2}^{*}}{\left(1+m x_{1}^{*}\right)^{2}} \\
\quad & {\left[2\left(\alpha d_{2}-r r_{1}\right)-\frac{a_{1} d_{2} y_{2}^{*}}{\left(1+m x_{1}^{*}\right)^{2}}\right] . }
\end{aligned}
$$

If $2\left(\alpha d_{2}-r r_{1}\right)>a_{1} d_{2} y_{2}^{*} /\left(1+m x_{1}^{*}\right)^{2}$, that is,

$\left(H_{3}\right)\left(r r_{1}-d_{2}\left(r_{1}+d_{1}\right)\right) / a d_{2}<2 d_{4}\left(r_{2}+d_{3}\right) /\left(a_{2} r_{2}-m d_{4}\left(r_{2}+\right.\right.$ $\left.\left.d_{3}\right)\right)+d_{4}\left(r_{2}+d_{3}\right) /\left(a_{2} r_{2}+m d_{4}\left(r_{2}+d_{3}\right)\right)$,

then (13) has no positive real roots. It is easy to check that $\left(\mathrm{H}_{2}\right)$ holds when $\left(\mathrm{H}_{3}\right)$ holds. Accordingly, by Theorem 3.4.1 in Kuang [8], we see that if $\left(H_{1}\right)$ and $\left(H_{3}\right)$ hold, then $E^{*}$ is locally asymptotically stable.

If the inequality in $\left(\mathrm{H}_{3}\right)$ is reversed, then (13) has a unique positive root $\omega_{0}$; that is, (8) has a pair of purely imaginary roots of the form $\pm i \omega_{0}$. Denote

$\tau_{k}$

$$
\begin{aligned}
& =\frac{2 k \pi}{\omega_{0}}+\frac{1}{\omega_{0}} \\
& \times \arccos \frac{\left(q_{2} \omega_{0}^{2}-q_{0}\right)\left(\omega_{0}^{4}-p_{2} \omega_{0}^{2}+p_{0}\right)+q_{1} \omega_{0}^{2}\left(p_{3} \omega_{0}^{2}-p_{1}\right)}{\left(q_{1} \omega_{0}\right)^{2}+\left(q_{2} \omega_{0}^{2}-q_{0}\right)^{2}}, \\
& k=0,1,2 \ldots .
\end{aligned}
$$

By Theorem 3.4.1 in Kuang [8], we see that $E^{*}$ remains stable for $\tau<\tau_{0}$.

We now claim that

$$
\left.\frac{d(\operatorname{Re}(\lambda))}{d \tau}\right|_{\tau=\tau_{0}}>0
$$

This will show that there exists at least one eigenvalue with a positive real part for $\tau>\tau_{0}$. Moreover, the conditions for the existence of a Hopf bifurcation [10] are then satisfied 
yielding a periodic solution. To this end, differentiating (8) with respect to $\tau$, it follows that

$$
\begin{aligned}
\left(\frac{d \lambda}{d \tau}\right)^{-1}= & \frac{4 \lambda^{3}+3 p_{3} \lambda^{2}+2 p_{2} \lambda+p_{1}}{-\lambda\left(\lambda^{4}+p_{3} \lambda^{3}+p_{2} \lambda^{2}+p_{1} \lambda+p_{0}\right)} \\
& +\frac{2 q_{2} \lambda+q_{1}}{\lambda\left(q_{2} \lambda^{2}+q_{1} \lambda+q_{0}\right)}-\frac{\tau}{\lambda}
\end{aligned}
$$

Hence, a direct calculation shows that

$$
\begin{aligned}
& \operatorname{sgn}\left\{\frac{d(\operatorname{Re} \lambda)}{d \tau}\right\}_{\lambda=i \omega_{0}} \\
& =\operatorname{sgn}\left\{\operatorname{Re}\left(\frac{d \lambda}{d \tau}\right)^{-1}\right\}_{\lambda=i \omega_{0}} \\
& =\operatorname{sgn}\left\{\frac{\left(3 p_{3} \omega_{0}^{2}-p_{1}\right)\left(p_{3} \omega_{0}^{2}-p_{1}\right)+2\left(2 \omega_{0}^{2}-p_{2}\right)\left(\omega_{0}^{4}-p_{2} \omega_{0}^{2}+p_{0}\right)}{\omega_{0}^{2}\left(p_{1}-p_{3} \omega_{0}^{2}\right)^{2}+\left(\omega_{0}^{4}-p_{2} \omega_{0}^{2}+p_{0}\right)^{2}}\right. \\
& \left.+\frac{-q_{1}^{2}+2 q_{2} q_{0}-2 q_{2}^{2} \omega_{0}^{2}}{\left(q_{1} \omega_{0}\right)^{2}+\left(q_{2} \omega_{0}^{2}-q_{0}\right)^{2}}\right\} .
\end{aligned}
$$

We derive from (12) that

$$
\begin{gathered}
\omega_{0}^{2}\left(p_{1}-p_{3} \omega_{0}^{2}\right)^{2}+\left(\omega_{0}^{4}-p_{2} \omega_{0}^{2}+p_{0}\right)^{2} \\
=\left(q_{1} \omega_{0}\right)^{2}+\left(q_{2} \omega_{0}^{2}-q_{0}\right)^{2} .
\end{gathered}
$$

Hence it follows that

$$
\begin{aligned}
\operatorname{sgn}\left\{\frac{d(\operatorname{Re} \lambda)}{d \tau}\right\}_{\lambda=i \omega_{0}} \\
\quad=\operatorname{sgn}\left\{\frac{4 \omega_{0}^{6}+3 h_{3} \omega_{0}^{4}+2 h_{2} \omega_{0}^{2}+h_{1}}{\left(q_{1} \omega_{0}\right)^{2}+\left(q_{2} \omega_{0}^{2}-q_{0}\right)^{2}}\right\}>0 .
\end{aligned}
$$

Therefore, the transversal condition holds and a Hopf bifurcation occurs at $\omega=\omega_{0}, \tau=\tau_{0}$.

In conclusion, we have the following results.

Theorem 1. For system (2), one has the following.

(i) If $r r_{1}<d_{2}\left(r_{1}+d_{1}\right)$, then the trivial equilibrium $E_{0}(0,0,0,0$,$) is locally asymptotically stable; if r r_{1}>$ $d_{2}\left(r_{1}+d_{1}\right)$, then $E_{0}$ is unstable.

(ii) If $0<\left(r r_{1}-d_{2}\left(r_{1}+d_{1}\right)\right) / a d_{2}<d_{4}\left(r_{2}+d_{3}\right) /\left(a_{2} r_{2}-\right.$ $\left.\mathrm{md}_{4}\left(r_{2}+d_{3}\right)\right)$, then the predator-extinction equilibrium $E_{1}\left(x_{1}^{+}, x_{2}^{+}, 0,0\right)$ is locally asymptotically stable;

if $\left(r r_{1}-d_{2}\left(r_{1}+d_{1}\right)\right) / a d_{2}>d_{4}\left(r_{2}+d_{3}\right) /\left(a_{2} r_{2}-m d_{4}\left(r_{2}+\right.\right.$ $\left.\left.d_{3}\right)\right)>0$, then $E_{1}$ is unstable.

(iii) Let $\left(H_{1}\right)$ hold. If $\left(H_{3}\right)$ holds, then the coexistence equilibrium $E^{*}\left(x_{1}^{*}, x_{2}^{*}, y_{1}^{*}, y_{2}^{*}\right)$ is locally asymptotically stable for all $\tau \geq 0$; if $\left(\mathrm{H}_{2}\right)$ holds and the inequality in $\left(\mathrm{H}_{3}\right)$ is reversed, then there exists a positive number $\tau_{0}$, such that $E^{*}$ is locally asymptotically stable if $0<$ $\tau<\tau_{0}$ and is unstable if $\tau>\tau_{0}$. Further, system (2) undergoes a Hopf bifurcation at $E^{*}$ when $\tau=\tau_{0}$.
We now give an example to illustrate the main results in Theorem 1 .

Example 2. In (2), let $a=16, a_{1}=16, a_{2}=3, d_{1}=1 / 8$, $d_{2}=1 / 2, d_{3}=1 / 8, d_{4}=1 / 8, r=5, r_{1}=1, r_{2}=1$, and $m=1 / 10$. It is easy to show that $\left(r r_{1}-d_{2}\left(r_{1}+d_{1}\right)\right) / a d_{2} \approx$ 0.5547 and $d_{4}\left(r_{2}+d_{3}\right) /\left(a_{2} r_{2}-m d_{4}\left(r_{2}+d_{3}\right)\right) \approx 0.0471$; that is, the condition $\left(H_{1}\right)$ holds. Hence, system $(2)$ has a unique coexistence equilibrium $E^{*}(0.0471,0.0942,0.0637,0.5100)$. By calculation, we have $p_{3}\left(p_{2}+q_{2}\right)-\left(p_{1}+q_{1}\right) \approx 177.6328>0$, $p_{3}\left(p_{2}+q_{2}\right)\left(p_{1}+q_{1}\right)-\left(p_{1}+q_{1}\right)^{2}-p_{3}^{2}\left(p_{0}+q_{0}\right) \approx 193.0344>0$, $2\left(\alpha d_{2}-r r_{1}\right)-a_{1} d_{2} y_{2}^{*} /\left(1+m x_{1}^{*}\right)^{2} \approx-3.3262<0$ and $\tau_{0} \approx 2.3729$. By Theorem $1, E^{*}$ is locally asymptotically stable if $0<\tau<\tau_{0}$ and is unstable if $\tau>\tau_{0}$, and system (2) undergoes a Hopf bifurcation at $E^{*}$ when $\tau=\tau_{0}$. Numerical simulation illustrates this fact (see Figure 1).

\section{Permanence}

In this section, we are concerned with the permanence of system (2).

Definition 3. System (2) is said to be permanent if there are positive constants $m_{1}, m_{2}, M_{1}$, and $M_{2}$, such that each positive solution of system (2) satisfies

$$
\begin{aligned}
& m_{1} \leq \liminf _{t \rightarrow+\infty} x_{i}(t) \leq \limsup _{t \rightarrow+\infty} x_{i}(t) \leq M_{1}, \quad i=1,2, \\
& m_{2} \leq \liminf _{t \rightarrow+\infty} y_{i}(t) \leq \limsup _{t \rightarrow+\infty} y_{i}(t) \leq M_{2}, \quad i=1,2 .
\end{aligned}
$$

Lemma 4. There are positive constants $M_{1}$ and $M_{2}$, such that, for any positive solution $\left(x_{1}(t), x_{2}(t), y_{1}(t), y_{2}(t)\right)$ of system $(2)$,

$$
\limsup _{t \rightarrow+\infty} x_{i}(t) \leq M_{1}, \quad \limsup _{t \rightarrow+\infty} y_{i}(t) \leq M_{2}, \quad i=1,2 .
$$

Proof. Let $\left(x_{1}(t), x_{2}(t), y_{1}(t), y_{2}(t)\right)$ be any positive solution of system (2) with initial conditions (3). Define

$$
V(t)=x_{1}(t-\tau)+\frac{r+d_{2}}{d_{2}} x_{2}(t-\tau)+\frac{a_{1}}{a_{2}} y_{1}(t)+\frac{a_{1}}{a_{2}} y_{2}(t) \text {. }
$$

Calculating the derivative of $V(t)$ along positive solutions of system (2), it follows that

$$
\begin{aligned}
\dot{V}(t)= & -d_{1} x_{1}(t-\tau)-d_{2} x_{2}(t-\tau)-\frac{a_{1}}{a_{2}} d_{3} y_{1}(t) \\
& -\frac{a_{1}}{a_{2}} d_{4} y_{2}(t)+\frac{r r_{1}}{d_{2}} x_{1}(t-\tau)-a x_{1}^{2}(t-\tau) \\
\leq & -d V(t)-a\left(x_{1}(t-\tau)-\frac{r r_{1}}{2 a d_{2}}\right)^{2}+\frac{\left(r r_{1}\right)^{2}}{4 a d_{2}^{2}} \\
\leq & -d V(t)+\frac{\left(r r_{1}\right)^{2}}{4 a d_{2}^{2}},
\end{aligned}
$$




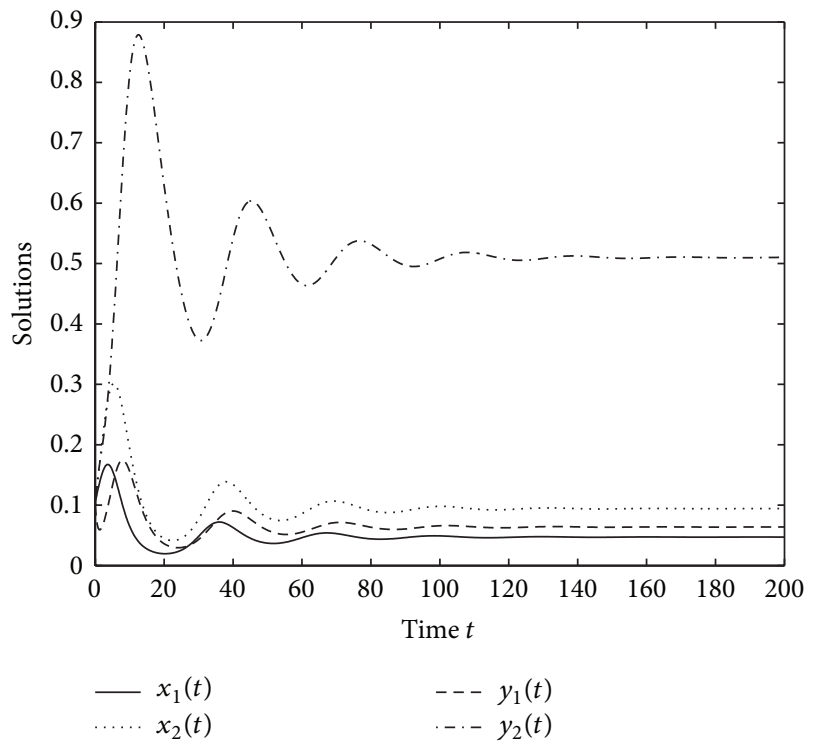

(a)

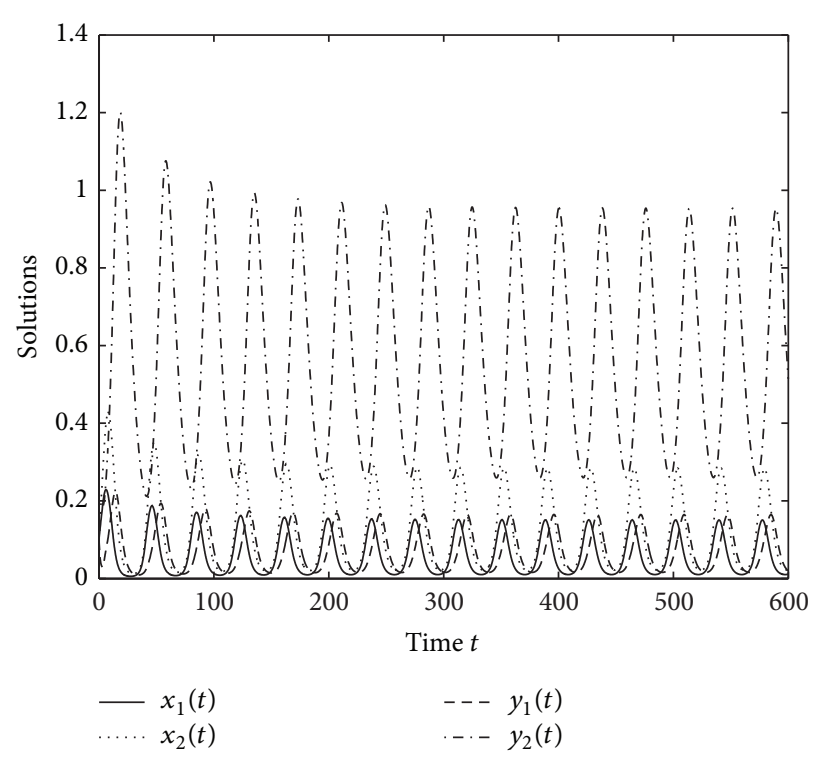

(b)

FiguRE 1: The temporal solution found by numerical integration of system (2) with $\tau=0.1$ and $\tau=3$, respectively; $\left(\phi_{1}, \phi_{2}, \varphi_{1}, \varphi_{2}\right)=$ $(0.1,0.1,0.1,0.1)$.

where $d=\min \left\{d_{1}, d_{2}^{2} /\left(r+d_{2}\right), d_{3}, d_{4}\right\}$. This inequality yields $\lim \sup _{t \rightarrow+\infty} V(t) \leq\left(r r_{1}\right)^{2} /\left(4 a d d_{2}^{2}\right)$. If we choose $M_{1}=$ $\left(r r_{1}\right)^{2} /\left(4 a d d_{2}\left(r+d_{2}\right)\right)$ and $M_{2}=a_{2}\left(r r_{1}\right)^{2} /\left(4 a a_{1} d d_{2}^{2}\right)$, then (22) follows. This completes the proof.

In order to study the permanence of system (2), we refer to persistence theory on infinite dimensional systems developed by Hale and Waltman in [11].

Let $X$ be a complete metric space with metric $d$. Suppose that $T:[0,+\infty] \times X \rightarrow X$ is a continuous map with the following properties:

$$
T_{t} \circ T_{s}=T_{t+s}, \quad t, s \geq 0, \quad T_{0}(x)=x, \quad x \in X,
$$

where $T_{t}$ denotes the mapping from $X$ to $X$ given by $T_{t}(x)=$ $T(t, x)$. The distance $d(x, Y)$ of a point $x \in X$ from a subset $Y$ of $X$ is defined by $d(x, Y)=\inf _{y \in Y} d(x, y)$. Recall that the positive orbit $\gamma^{+}(x)=\bigcup_{t \geq 0}\{T(t) x\}$, and its $\omega$-limit set is $\omega(x)=\bigcap_{s \geq 0} \bigcup_{t \geq s}\{\bar{T}(t) x\}$. Define $W^{s}(A)$, the strong stable set of a compact invariant set $A$, to be $W^{s}(A)=\{x: x \in$ $X, \omega(x) \neq \varnothing, \omega(x) \subset A\}$.

(A1) Assume that $X^{0}$ is open and dense in $X$ and $X^{0} \cup X_{0}=X, X^{0} \cap X_{0}=\varnothing$. Moreover, the $C^{0}$ semigroup $T(t)$ on $X$ satisfies

$$
T(t): X^{0} \longrightarrow X^{0}, \quad T(t): X_{0} \longrightarrow X_{0} .
$$

Let $T_{b}(t)=\left.T(t)\right|_{X_{0}}$ and $A_{b}$ be the global attractor for $T_{b}(t)$. Define $\widetilde{A}_{b}=\bigcup_{x \in A_{b}} \omega(x)$.

Lemma 5 (Hale and Waltman [11]). Suppose that $T(t)$ satisfies (A1) and the following conditions:

(i) there is a $t_{0} \geq 0$ such that $T(t)$ is compact for $t>t_{0}$; (ii) $T(t)$ is point dissipative in $X$;

(iii) $\bar{A}_{b}$ is isolated and has an acyclic covering $\widehat{M}$, where $\widehat{M}=\left\{\widehat{M}_{1}, \widehat{M}_{2}, \ldots, \widehat{M}_{n}\right\} ;$

(iv) $W^{s}\left(\widehat{M}_{i}\right) \cap X^{0}=\varnothing$ for $i=1,2, \ldots, n$.

Then $X_{0}$ is a uniform repeller with respect to $X^{0}$; that is, there is an $\varepsilon>0$ such that, for any $x \in X^{0}, \liminf _{t \rightarrow+\infty} d(T(t) x$, $\left.X_{0}\right) \geq \varepsilon$.

We are now able to state and prove the result on the permanence of system (2).

Theorem 6. If $\left(H_{1}\right)$ holds, then system (2) is permanent.

Proof. We need only to show that the boundaries of $R_{+0}^{4}$ repel positive solutions of system (2) uniformly. Let $C^{+}\left([-\tau, 0], R_{+0}^{4}\right)$ denote the space of continuous functions mapping $[-\tau, 0]$ into $R_{+0}^{4}$. Define

$$
\begin{aligned}
& C_{1}=\left\{\left(\phi_{1}, \phi_{2}, \varphi_{1}, \varphi_{2}\right) \in C^{+}\left([-\tau, 0], R_{+0}^{4}\right): \phi_{i}(\theta) \equiv 0,\right. \\
&\theta \in[-\tau, 0], i=1,2\}, \\
& C_{2}=\left\{\left(\phi_{1}, \phi_{2}, \varphi_{1}, \varphi_{2}\right) \in C^{+}\left([-\tau, 0], R_{+0}^{4}\right): \phi_{i}(\theta)>0,\right. \\
&\left.\varphi_{i}(\theta) \equiv 0, \theta \in[-\tau, 0], i=1,2\right\} .
\end{aligned}
$$

Denote $C_{0}=C_{1} \cup C_{2}$ and $C^{0}=\operatorname{int} C^{+}\left([-\tau, 0], R_{+0}^{4}\right)$.

In the following, we show that the conditions in Lemma 5 are satisfied. By the definition of $C^{0}$ and $C_{0}$, it is easy to see that $C^{0}$ and $C_{0}$ are positively invariant and the condition (ii) in Lemma 5 is clearly satisfied. Using the smoothing property of solutions of delay differential equations introduced in 
Kuang [8] (Theorem 2.2.8), it follows that condition (i) in Lemma 5 is satisfied. Thus, we need only to show that the conditions (iii) and (iv) hold. Clearly, corresponding to $x_{i}(t)=y_{i}(t)=0$ and $x_{1}(t)=x_{1}^{+}, x_{2}(t)=x_{2}^{+}, y_{i}(t)=0$, respectively, there are two constant solutions in $C_{0}: \widetilde{E}_{0} \in C_{1}$ and $\widetilde{E}_{1} \in C_{2}$ satisfying

$$
\begin{gathered}
\widetilde{E}_{0}=\left\{\left(\phi_{1}, \phi_{2}, \varphi_{1}, \varphi_{2}\right) \in C^{+}\left([-\tau, 0], R_{+0}^{4}\right): \phi_{i}(\theta) \equiv 0,\right. \\
\left.\varphi_{i}(\theta) \equiv 0, \theta \in[-\tau, 0]\right\} \\
\widetilde{E}_{1}=\left\{\left(\phi_{1}, \phi_{2}, \varphi_{1}, \varphi_{2}\right) \in C^{+}\left([-\tau, 0], R_{+0}^{4}\right): \phi_{1}(\theta)=x_{1}^{+},\right. \\
\left.\phi_{2}(\theta)=x_{2}^{+}, \varphi_{i}(\theta) \equiv 0, \theta \in[-\tau, 0]\right\} .
\end{gathered}
$$

We now verify the condition (iii) in Lemma 5. If $\left(x_{1}(t), x_{2}(t)\right.$, $\left.y_{1}(t), y_{2}(t)\right)$ is a solution of system (2) initiating from $C_{1}$, then $\dot{y}_{1}(t)=-\left(d_{3}+r_{2}\right) y_{1}(t)$ and $\dot{y}_{2}(t)=r_{2} y_{1}(t)-d_{4} y_{2}(t)$, which yields $\lim _{t \rightarrow+\infty} y_{i}(t)=0, i=1,2$. If $\left(x_{1}(t), x_{2}(t), y_{1}(t), y_{2}(t)\right)$ is a solution of system (2) initiating from $C_{2}$ with $\phi_{i}(0)>0$, then it follows from the first and second equations of system (2) that $\dot{x}_{1}(t)=r x_{2}(t)-\left(r_{1}+d_{1}\right) x_{1}(t)-a x_{1}^{2}(t)$ and $\dot{x}_{2}(t)=$ $r_{1} x_{1}(t)-d_{2} x_{2}(t)$. If $r r_{1}>d_{2}\left(r_{1}+d_{1}\right)$ holds, then $x_{1}(t) \rightarrow x_{1}^{+}$, $x_{2}(t) \rightarrow x_{2}^{+}$as $t \rightarrow+\infty$. Noting that $C_{1} \cap C_{2}=\varnothing$, we see that the invariant sets $\widetilde{E}_{0}$ and $\widetilde{E}_{1}$ are isolated. Hence, $\left\{\widetilde{E}_{0}, \widetilde{E}_{1}\right\}$ is isolated and is an acyclic covering satisfying the condition (iii) in Lemma 5.

We now verify that $W^{s}\left(\widetilde{E}_{0}\right) \bigcap C^{0}=\varnothing$ and $W^{s}\left(\widetilde{E}_{1}\right) \bigcap C^{0}=$ $\varnothing$. Here, we only prove the second equation since the proof of the first equation is simple. Assume $W^{s}\left(\widetilde{E}_{1}\right) \bigcap C^{0} \neq \varnothing$. Then there is a positive solution $\left(x_{1}(t), x_{2}(t), y_{1}(t), y_{2}(t)\right)$ satisfying

$$
\lim _{t \rightarrow+\infty}\left(x_{1}(t), x_{2}(t), y_{1}(t), y_{2}(t)\right)=\left(x_{1}^{+}, x_{2}^{+}, 0,0\right) \text {. }
$$

Hence, for $\varepsilon>0$ sufficiently small, there is a $t_{0}>0$ such that, if $t>t_{0}, x_{1}^{+}-\varepsilon<x_{2}(t)<x_{1}^{+}+\varepsilon$.

Since $\left(H_{1}\right)$ holds, we can choose $\varepsilon>0$ sufficiently small, such that

$$
\frac{a_{2} r_{2}\left(x_{1}^{+}-\varepsilon\right)}{1+m\left(x_{1}^{+}-\varepsilon\right)}>d_{4}\left(r_{2}+d_{3}\right) \text {. }
$$

For $\varepsilon>0$ sufficiently small satisfying (30), it follows from the third and the fourth equations of system (2) that, for $t>t_{0}+\tau$,

$$
\begin{gathered}
\dot{y}_{1}(t) \geq \frac{a_{2}\left(x_{1}^{+}-\varepsilon\right)}{1+m\left(x_{1}^{+}-\varepsilon\right)} y_{2}(t-\tau)-\left(r_{2}+d_{3}\right) y_{2}(t), \\
\dot{y}_{2}(t)=r_{2} y_{1}(t)-d_{4} y_{1}(t) .
\end{gathered}
$$

Define

$$
A_{\epsilon}=\left(\begin{array}{cc}
-\left(r_{2}+d_{3}\right) & \frac{a_{2}\left(x_{1}^{+}-\varepsilon\right)}{1+m\left(x_{1}^{+}-\varepsilon\right)} \\
r_{2} & -d_{4}
\end{array}\right) .
$$

Since $A_{\epsilon}$ has positive off-diagonal elements, by the PerronFrobenius theorem, there is a positive eigenvector $\eta$ for the maximum eigenvalue $\mu$ of $A_{\epsilon}$. Noting that (30) holds, a direct calculation shows that $\mu>0$. Using a similar argument as that in the proof of Theorem 2.1 in [2], one can show that $\lim _{t \rightarrow+\infty} y_{i}(t)=+\infty(i=1,2)$. This contradicts Lemma 4. Hence, we have $W^{s}\left(\widetilde{E}_{1}\right) \cap C^{0}=\varnothing$. By Lemma 5, we conclude that $C_{0}$ repels positive solutions of system (2) uniformly. Therefore, system (2) is permanent. The proof is complete.

\section{Global Stability}

In this section, we are concerned with the global stability of each of the feasible equilibria of system (2). The strategy of proofs is to use Lyapunov functionals and the LaSalle invariant principle.

Theorem 7. If $r r_{1}<d_{2}\left(r_{1}+d_{1}\right)$, then the trivial equilibrium $E_{0}(0,0,0,0)$ of system (2) is globally asymptotically stable.

Proof. Let $\left(x_{1}(t), x_{2}(t), y_{1}(t), y_{2}(t)\right)$ be any positive solution of system (2) with initial conditions (3). By Theorem 1, we see that if $r r_{1}<d_{2}\left(r_{1}+d_{1}\right)$, then $E_{0}$ is locally asymptotically stable. Define

$$
\begin{aligned}
V_{1}(t)= & x_{1}(t)+\frac{r}{d_{2}} x_{2}(t)+\frac{a_{1}}{a_{2}} y_{1}(t)+\frac{a_{1}\left(r_{2}+d_{3}\right)}{a_{2} r_{2}} y_{2}(t) \\
& +a_{1} \int_{t-\tau}^{t} \frac{x_{1}(s) y_{2}(s)}{1+m x_{1}(s)} d s .
\end{aligned}
$$

Calculating the derivative of $V_{1}(t)$ along positive solutions of system (2), it follows that

$$
\begin{aligned}
\dot{V}_{1}(t)= & \dot{x}_{1}(t)+\frac{r}{d_{2}} \dot{x}_{2}(t)+\frac{a_{1}}{a_{2}} \dot{y}_{1}(t)+\frac{a_{1}\left(r_{2}+d_{3}\right)}{a_{2} r_{2}} \dot{y}_{2}(t) \\
& +\frac{a_{1} x_{1}(t) y_{2}(t)}{1+m x_{1}(t)}-\frac{a_{1} x_{1}(t-\tau) y_{2}(t-\tau)}{1+m x_{1}(t-\tau)} \\
= & \frac{r r_{1}-d_{2}\left(r_{1}+d_{1}\right)}{d_{2}} x_{1}(t)-a x_{1}^{2}(t) \\
& -\frac{a_{1} d_{4}\left(r_{2}+d_{3}\right)}{a_{2} r_{2}} y_{2}(t) .
\end{aligned}
$$

If $r r_{1}<d_{2}\left(r_{1}+d_{1}\right)$, it then follows from (34) that $\dot{V}_{1}(t) \leq 0$. By Theorem 5.3.1 in [10], solutions approach $M$, the largest invariant subset of $\left\{\dot{V}_{1}(t)=0\right\}$. Clearly, we see from (34) that $\dot{V}_{1}(t)=0$ if and only if $x_{1}(t)=0, y_{2}(t)=0$. Noting that $M$ is invariant, for each element in $M$, we have $x_{1}(t)=0, y_{2}(t)=0$. It therefore follows from the second and fourth equations of system (2) that

$$
0=\dot{x}_{1}(t)=r x_{2}(t), \quad 0=\dot{y}_{2}(t)=r_{2} y_{1}(t),
$$

which yields $x_{2}(t)=0, y_{1}(t)=0$. Hence, $\dot{V}_{1}(t)=0$ if and only if $\left(x_{1}(t), x_{2}(t), y_{1}(t), y_{2}(t)\right)=(0,0,0,0)$. Accordingly, the global asymptotic stability of $E_{0}$ follows from LaSalle's invariant principle. This completes the proof. 
Theorem 8. The predator-extinction equilibrium $E_{1}\left(x_{1}^{+}, x_{2}^{+}\right.$, $0,0)$ of system (2) is globally asymptotically stable provided that

$$
\begin{aligned}
\left(H_{4}\right) & 0<\left(r r_{1}-d_{2}\left(r_{1}+d_{1}\right)\right) / a d_{2}<d_{4}\left(r_{2}+d_{3}\right) /\left(a_{2} r_{2}-\right. \\
& \left.m d_{4}\left(r_{2}+d_{3}\right)\right) .
\end{aligned}
$$

Proof. Assume that $\left(x_{1}(t), x_{2}(t), y_{1}(t), y_{2}(t)\right)$ is any positive solution of system (2) with initial conditions (3). By Theorem 1, we see that if $\left(H_{4}\right)$ holds, then $E_{1}$ is locally asymptotically stable. System (2) can be rewritten as

$$
\begin{gathered}
\dot{x}_{1}(t)=\frac{r}{x_{1}^{+}}\left[-x_{2}(t)\left(x_{1}(t)-x_{1}^{+}\right)+x_{1}(t)\left(x_{2}(t)-x_{2}^{+}\right)\right] \\
+x_{1}(t)\left[-a\left(x_{1}(t)-x_{1}^{+}\right)\right]-\frac{a_{1} x_{1}(t) y_{2}(t)}{1+m x_{1}(t)}, \\
\dot{x}_{2}(t)=\frac{r_{1}}{x_{2}^{+}}\left[-x_{1}(t)\left(x_{2}(t)-x_{2}^{+}\right)+x_{2}(t)\left(x_{1}(t)-x_{1}^{+}\right)\right], \\
\dot{y}_{1}(t)=\frac{a_{2} x_{1}(t-\tau) y_{2}(t-\tau)}{1+m x_{1}(t-\tau)}-\left(r_{2}+d_{3}\right) y_{1}(t), \\
\dot{y}_{2}(t)=r_{2} y_{1}(t)-d_{4} y_{2}(t) .
\end{gathered}
$$

Define

$$
\begin{aligned}
V_{21}(t)= & x_{1}-x_{1}^{+}-x_{1}^{+} \ln \frac{x_{1}}{x_{1}^{+}}+c_{1}\left(x_{2}-x_{2}^{+}-x_{2}^{+} \ln \frac{x_{2}}{x_{2}^{+}}\right) \\
& +k_{1} y_{1}+k_{2} y_{2}
\end{aligned}
$$

where $c_{1}=r x_{2}^{+} /\left(r_{1} x_{1}^{+}\right), k_{1}=a_{1}\left(1+m x_{1}^{+}\right) / a_{2}$, and $k_{2}=\left(r_{2}+\right.$ $\left.d_{3}\right) k_{1} / r_{2}$. Calculating the derivative of $V_{21}(t)$ along positive solutions of system (2), it follows that

$$
\begin{aligned}
\dot{V}_{21}(t) & \frac{r\left(x_{1}(t)-x_{1}^{+}\right)}{x_{1}^{+} x_{1}(t)} \\
& \times\left[-x_{2}(t)\left(x_{1}(t)-x_{1}^{+}\right)+x_{1}(t)\left(x_{2}(t)-x_{2}^{+}\right)\right] \\
& -a\left(x_{1}(t)-x_{1}^{+}\right)^{2}-\frac{\left(x_{1}(t)-x_{1}^{+}\right) a_{1} x_{1}(t) y_{2}(t)}{x_{1}(t)\left(1+m x_{1}(t)\right)} \\
& +\frac{r\left(x_{2}(t)-x_{2}^{+}\right)}{x_{1}^{+} x_{2}(t)} \\
& \times\left[-x_{1}(t)\left(x_{2}(t)-x_{2}^{+}\right)+x_{2}(t)\left(x_{1}(t)-x_{1}^{+}\right)\right] \\
& +\frac{a_{2} k_{1} x_{1}(t-\tau) y_{2}(t-\tau)}{1+m x_{1}(t-\tau)}-k_{2} d_{4} y_{2}(t) \\
= & -\frac{r}{x_{1}^{+}}\left[\sqrt{\frac{x_{2}(t)}{x_{1}(t)}}\left(x_{1}(t)-x_{1}^{+}\right)-\sqrt{\frac{x_{1}(t)}{x_{2}(t)}}\left(x_{2}(t)-x_{2}^{+}\right)\right]^{2} \\
& -a\left(x_{1}(t)-x_{1}^{+}\right)^{2}
\end{aligned}
$$

$$
\begin{aligned}
& -a_{1}\left(1+m x_{1}^{+}\right)\left[\frac{x_{1}(t) y_{2}(t)}{1+m x_{1}(t)}-\frac{x_{1}(t-\tau) y_{2}(t-\tau)}{1+m x_{1}(t-\tau)}\right] \\
& +\left(a_{1} x_{1}^{+}-k_{2} d_{4}\right) y_{2}(t) .
\end{aligned}
$$

Define

$$
V_{2}(t)=V_{21}(t)+a_{1}\left(1+m x_{1}^{+}\right) \int_{t-\tau}^{t} \frac{x_{1}(s) y_{2}(s)}{1+m x_{1}(s)} d s
$$

We derive from (38) and (39) that

$$
\begin{aligned}
\dot{V}_{2}(t)=-\frac{r}{x_{1}^{+}}[ & \sqrt{\frac{x_{2}(t)}{x_{1}(t)}}\left(x_{1}(t)-x_{1}^{+}\right) \\
& \left.-\sqrt{\frac{x_{1}(t)}{x_{2}(t)}}\left(x_{2}(t)-x_{2}^{+}\right)\right]^{2} \\
& -a\left(x_{1}(t)-x_{1}^{+}\right)^{2}-\left(k_{2} d_{4}-a_{1} x_{1}^{+}\right) y_{2}(t) .
\end{aligned}
$$

If $\left(\mathrm{H}_{4}\right)$ holds, it then follows from $(40)$ that $\dot{V}_{2}(t) \leq 0$. By Theorem 5.3.1 in [10], solutions approach $M$, the largest invariant subset of $\left\{\dot{V}_{2}(t)=0\right\}$. Clearly, we see from (40) that $\dot{V}_{2}(t)=0$ with equality if only if $x_{1}=x_{1}^{+}, x_{2}=x_{2}^{+}$, and $y_{2}=0$. It follows from the fourth equation of system (2) that $0=\dot{y}_{2}(t)=r_{2} y_{1}(t)$, which yields $y_{1}=0$. Hence, $\dot{V}_{2}(t)=0$ if only if $x_{1}=x_{1}^{+}, x_{2}=x_{2}^{+}, y_{1}=0$, and $y_{2}=0$. Using the LaSalle invariant principle, the global asymptotic stability of $E_{1}$ follows. This completes the proof.

Theorem 9. The coexistence equilibrium $E^{*}\left(x_{1}^{*}, x_{2}^{*}, y_{1}^{*}, y_{2}^{*}\right)$ of system (2) is globally asymptotically stable provided that

$$
\begin{gathered}
\left(H_{5}\right) \underset{x_{1}}{\left.m d_{4}\left(r_{2}+d_{3}\right)\right) .} .\left(r r_{1}-d_{2}\left(r_{1}+d_{1}\right)\right) / a d_{2}-d_{4}\left(r_{2}+d_{3}\right) /\left(a_{2} r_{2}-\right. \\
\end{gathered}
$$

Here, $\underline{x}_{1}$ is the uniform persistency constant for $x_{1}$ satisfying $\liminf _{t \rightarrow \infty} x_{1}(t) \geq \underline{x}_{1}$.

Proof. Let $\left(x_{1}(t), x_{2}(t), y_{1}(t), y_{2}(t)\right)$ be any positive solution of system (2) with initial conditions (3). Since $\left(H_{5}\right)$ holds, there is a $t_{1}>0$, such that

$$
x_{1}(t)>\frac{r r_{1}-d_{2}\left(r_{1}+d_{1}\right)}{a d_{2}}-\frac{d_{4}\left(r_{2}+d_{3}\right)}{a_{2} r_{2}-m d_{4}\left(r_{2}+d_{3}\right)}
$$

for all $t \geq t_{1}$. Accordingly, we have

$$
x_{1}^{*}>\frac{r r_{1}-d_{2}\left(r_{1}+d_{1}\right)}{a d_{2}}-\frac{d_{4}\left(r_{2}+d_{3}\right)}{a_{2} r_{2}-m d_{4}\left(r_{2}+d_{3}\right)} .
$$

In this case, it is easy to show that $\left(H_{1}\right)$ and $\left(H_{3}\right)$ hold. By Theorem $1, E^{*}$ is locally asymptotically stable for all $\tau>0$. 
System (2) can be rewritten as

$$
\begin{gathered}
\dot{x}_{1}(t)=\frac{r}{x_{1}^{*}}\left[-x_{2}(t)\left(x_{1}(t)-x_{1}^{*}\right)+x_{1}(t)\left(x_{2}(t)-x_{2}^{*}\right)\right] \\
\quad+x_{1}(t)\left[-a\left(x_{1}(t)-x_{1}^{*}\right)\right] \\
\quad+\frac{a_{1} y_{2}^{*}}{1+m x_{1}^{*}} x_{1}(t)-\frac{a_{1} x_{1}(t) y_{2}(t)}{1+m x_{1}(t)}, \\
\dot{x}_{2}(t)=\frac{r_{1}}{x_{2}^{*}}\left[-x_{1}(t)\left(x_{2}(t)-x_{2}^{*}\right)+x_{2}(t)\left(x_{1}(t)-x_{1}^{*}\right)\right], \\
\dot{y}_{1}(t)=\frac{a_{2} x_{1}(t-\tau) y_{2}(t-\tau)}{1+m x_{1}(t-\tau)}-\left(r_{2}+d_{3}\right) y_{1}(t), \\
\dot{y}_{2}(t)=r_{2} y_{1}(t)-d_{4} y_{2}(t) .
\end{gathered}
$$

Define

$$
\begin{aligned}
V_{31}(t)= & x_{1}-x_{1}^{*}-x_{1}^{*} \ln \frac{x_{1}}{x_{1}^{*}}+c_{1}\left(x_{2}-x_{2}^{*}-x_{2}^{*} \ln \frac{x_{2}}{x_{2}^{*}}\right) \\
& +k_{1}\left(y_{1}-y_{1}^{*}-y_{1}^{*} \ln \frac{y_{1}}{y_{1}^{*}}\right) \\
& +k_{2}\left(y_{2}-y_{2}^{*}-y_{2}^{*} \ln \frac{y_{2}}{y_{2}^{*}}\right)
\end{aligned}
$$

where $c_{1}=r x_{2}^{*} /\left(r_{1} x_{1}^{*}\right), k_{1}=a_{1}\left(1+m x_{1}^{*}\right) / a_{2}, k_{2}=k_{1}\left(r_{2}+\right.$ $\left.d_{3}\right) / r_{2}$. Calculating the derivative of $V_{31}(t)$ along positive solutions of system (2), it follows that

$$
\begin{aligned}
\dot{V}_{31}(t) & x_{1}(t)-x_{1}^{*} \dot{x}_{1}(t)+c_{1} \frac{x_{2}(t)-x_{2}^{*}}{x_{1}(t)} \dot{x}_{2}(t) \\
& +k_{1} \frac{y_{1}(t)-y_{1}^{*}}{y_{1}(t)} \dot{y}_{1}(t)+\frac{y_{2}(t)-y_{2}^{*}}{y_{2}(t)} \dot{y}_{2}(t) \\
= & \frac{r\left(x_{1}(t)-x_{1}^{*}\right)}{x_{1}^{*} x_{1}(t)} \\
& \times\left[-x_{2}(t)\left(x_{1}(t)-x_{1}^{*}\right)+x_{1}(t)\left(x_{2}(t)-x_{2}^{*}\right)\right] \\
& -a\left(x_{1}(t)-x_{1}^{*}\right)^{2}+\frac{a_{1} y_{2}^{*}\left(x_{1}(t)-x_{1}^{*}\right)}{1+m x_{1}^{*}} \\
& -\frac{a_{1} y_{2}(t)\left(x_{1}(t)-x_{1}^{*}\right)}{1+m x_{1}(t)}+\frac{r\left(x_{2}(t)-x_{2}^{*}\right)}{x_{1}^{*} x_{2}(t)} \\
& \times\left[-x_{1}(t)\left(x_{2}(t)-x_{2}^{*}\right)+x_{2}(t)\left(x_{1}(t)-x_{1}^{*}\right)\right] \\
& +\frac{a_{2} k_{1}\left(y_{1}(t)-y_{1}^{*}\right) x_{1}(t-\tau) y_{2}(t-\tau)}{y_{1}(t)\left(1+m x_{1}(t-\tau)\right)} \\
& +k_{2} r_{2} \frac{y_{1}(t)}{y_{2}(t)}\left(y_{2}(t)-y_{2}^{*}\right) \\
& -k_{1}\left(r_{2}+d_{3}\right)\left(y_{1}(t)-y_{1}^{*}\right)-k_{2} d_{4}\left(y_{2}(t)-y_{2}^{*}\right)
\end{aligned}
$$

$$
\begin{aligned}
= & -\frac{r}{x_{1}^{*}}\left[\sqrt{\frac{x_{2}(t)}{x_{1}(t)}}\left(x_{1}(t)-x_{1}^{*}\right)-\sqrt{\frac{x_{1}(t)}{x_{2}(t)}}\left(x_{2}(t)-x_{2}^{*}\right)\right]^{2} \\
& -a_{1}\left(1+m x_{1}^{*}\right) \frac{y_{1}^{*} x_{1}(t-\tau) y_{2}(t-\tau)}{y_{1}(t)\left(1+m x_{1}(t-\tau)\right)} \\
& -a_{1}\left(1+m x_{1}^{*}\right) \frac{x_{1}(t) y_{2}(t)}{1+m x_{1}(t)} \\
& +a_{2} k_{1} \frac{x_{1}(t-\tau) y_{2}(t-\tau)}{1+m x_{1}(t-\tau)} \\
& +\frac{a_{1} y_{2}^{*}}{1+m x_{1}^{*}}\left(x_{1}(t)-x_{1}^{*}\right)-k_{2} r_{2} y_{2}^{*} \frac{y_{1}(t)}{y_{2}(t)} \\
& -a\left(x_{1}(t)-x_{1}^{*}\right)^{2}+k_{1}\left(r_{2}+d_{3}\right) y_{1}^{*}+k_{2} d_{4} y_{2}^{*} .
\end{aligned}
$$

Define

$$
\begin{aligned}
V_{3}(t) & \\
=V_{31}(t)+a_{2} k_{1} \int_{t-\tau}^{t} & {\left[\frac{x_{1}(s) y_{2}(s)}{1+m x_{1}(s)}-\frac{x_{1}^{*} y_{2}^{*}}{1+m x_{1}^{*}}-\frac{x_{1}^{*} y_{2}^{*}}{1+m x_{1}^{*}}\right.} \\
& \left.\times \ln \frac{\left(1+m x_{1}^{*}\right) x_{1}(s) y_{2}(s)}{x_{1}^{*} y_{2}^{*}\left(1+m x_{1}(s)\right)}\right] d s .
\end{aligned}
$$

We derive from (45) and (46) that

$\dot{V}_{3}(t)$

$$
\begin{aligned}
& =-\frac{r}{x_{1}^{*}}\left[\sqrt{\frac{x_{2}(t)}{x_{1}(t)}}\left(x_{1}(t)-x_{1}^{*}\right)-\sqrt{\frac{x_{1}(t)}{x_{2}(t)}}\left(x_{2}(t)-x_{2}^{*}\right)\right]^{2} \\
& -a_{1} x_{1}^{*} y_{2}^{*}\left[\frac{y_{2}^{*} y_{1}(t)}{y_{1}^{*} y_{2}(t)}-1-\ln \frac{y_{2}^{*} y_{1}(t)}{y_{1}^{*} y_{2}(t)}\right] \\
& -a_{1} x_{1}^{*} y_{2}^{*}\left[\frac{y_{1}^{*}\left(1+m x_{1}^{*}\right) x_{1}(t-\tau) y_{2}(t-\tau)}{x_{1}^{*} y_{2}^{*} y_{1}(t)\left(1+m x_{1}(t-\tau)\right)}\right. \\
& \left.-1-\ln \frac{y_{1}^{*}\left(1+m x_{1}^{*}\right) x_{1}(t-\tau) y_{2}(t-\tau)}{x_{1}^{*} y_{2}^{*} y_{1}(t)\left(1+m x_{1}(t-\tau)\right)}\right] \\
& -a_{1} x_{1}^{*} y_{2}^{*}\left[\frac{x_{1}^{*}\left(1+m x_{1}(t)\right)}{x_{1}(t)\left(1+m x_{1}^{*}\right)}-1-\ln \frac{x_{1}^{*}\left(1+m x_{1}(t)\right)}{x_{1}(t)\left(1+m x_{1}^{*}\right)}\right] \\
& -\left(x_{1}(t)-x_{1}^{*}\right)^{2}\left[a-\frac{a_{1} y_{2}^{*}}{x_{1}(t)\left(1+m x_{1}^{*}\right)}\right] .
\end{aligned}
$$

If $\left(H_{5}\right)$ holds, for $t$ sufficiently enough, we have $a>$ $a_{1} y_{2}^{*} /\left(x_{1}(t)\left(1+m x_{1}^{*}\right)\right)$. This, together with (47), implies that $\dot{V}_{3}(t) \leq 0$, with equality if and only if

$$
\begin{gathered}
x_{1}=x_{1}^{*}, \quad x_{2}=x_{2}^{*}, \\
\frac{y_{2}^{*} y_{1}(t)}{y_{1}^{*} y_{2}(t)}=\frac{y_{1}^{*}\left(1+m x_{1}^{*}\right) x_{1}(t-\tau) y_{2}(t-\tau)}{x_{1}^{*} y_{2}^{*} y_{1}(t)\left(1+m x_{1}(t-\tau)\right)}=1 .
\end{gathered}
$$


We now look for the invariant subset $M$ within the set

$$
\begin{aligned}
M=\left\{\left(x_{1}, x_{2}, y_{1}, y_{2}\right): x_{1}=x_{1}^{*}, x_{2}=x_{2}^{*},\right. \\
\left.\quad \frac{y_{2}^{*} y_{1}(t)}{y_{1}^{*} y_{2}(t)}=\frac{y_{1}^{*}\left(1+m x_{1}^{*}\right) x_{1}(t-\tau) y_{2}(t-\tau)}{x_{1}^{*} y_{2}^{*} y_{1}(t)\left(1+m x_{1}(t-\tau)\right)}=1\right\} .
\end{aligned}
$$

Since $x_{1}=x_{1}^{*}$ and $x_{2}=x_{2}^{*}$ on $M$ and consequently $0=$ $\dot{x}_{1}(t)=x_{1}^{*}\left[r r_{1} / d_{2}-\left(r_{1}+d_{1}\right)-a x_{1}^{*}-a_{1} y_{2}(t) /\left(1+m x_{1}^{*}\right)\right]$, which yields $y_{2}(t)=y_{2}^{*}$, it follows from the fourth equation of system (2) that $0=\dot{y}_{2}(t)=r_{2} y_{1}(t)-d_{4} y_{2}^{*}$, which leads to $y_{1}=y_{1}^{*}$. Hence, the only invariant set in $M$ is $M=$ $\left\{\left(x_{1}^{*}, x_{2}^{*}, y_{1}^{*}, y_{2}^{*}\right)\right\}$. Using the LaSalle invariant principle, the global asymptotic stability of $E^{*}$ follows. This completes the proof.

We give an example to illustrate the result in Theorem 9.

Example 10. In (2), let $a=160, a_{1}=5, a_{2}=3, d_{1}=1 / 8$, $d_{2}=1 / 4, d_{3}=1 / 8, d_{4}=1 / 8, r=2.2, r_{1}=1, r_{2}=1$, and $m=1 / 10$. It is easy to show that $\left(r r_{1}-d_{2}\left(r_{1}+d_{1}\right)\right) / a d_{2} \approx$ 0.0480 and $d_{4}\left(r_{2}+d_{3}\right) /\left(a_{2} r_{2}-m d_{4}\left(r_{2}+d_{3}\right)\right) \approx 0.0471$; that is, condition $\left(H_{1}\right)$ holds. Hence, system (2) has a unique coexistence equilibrium $E^{*}(0.0471,0.1884,0.0035,0.0281)$. Hence, by Theorem 6, system (2) is permanent. From the proof of Lemma 4, we have $\limsup _{t \rightarrow \infty} y_{2}(t) \leq M_{2}:=$ $a_{2} r^{2} /\left(4 a a_{1} d d_{2}^{2}\right) \approx 1.4520$. Hence, for $\varepsilon>0$ sufficiently small, there is a $t_{1}>0$ such that, if $t>t_{1}, y_{2}(t)<M_{2}+\varepsilon$. It follows from system (2) that, for $t>t_{1}$,

$$
\begin{gathered}
\dot{x}_{1}(t)>r x_{2}(t)-\left(r_{1}+d_{1}\right) x_{1}(t)-a x_{1}^{2}(t) \\
-a_{1}\left(M_{2}+\varepsilon\right) x_{1}(t) \\
\dot{x}_{2}(t)=r_{1} x_{1}(t)-d_{2} x_{2}(t),
\end{gathered}
$$

which yields

$$
\liminf _{t \rightarrow \infty} x_{1}(t) \geq \frac{r r_{1}-d_{2}\left(a_{1} M_{2}+r_{1}+d_{1}\right)}{a d_{2}}:=\underline{x_{1}} .
$$

By calculation, we derive that $x_{1} \approx 0.0026$ and $\left(r r_{1}-d_{2}\left(r_{1}+\right.\right.$ $\left.\left.d_{1}\right)\right) / a d_{2}-d_{4}\left(r_{2}+d_{3}\right) /\left(a_{2} r_{2}-m d_{4}\left(r_{2}+d_{3}\right)\right) \approx 0.00087$. By Theorem $9, E^{*}$ is globally asymptotically stable. Numerical simulation illustrates this fact (see Figure 2).

\section{Discussion}

In this paper, we have incorporated stage structure for both the predators and the prey into a predator-prey model with time delay due to the gestation of the predator and Holling type II functional response. By using Lyapunov functionals and the LaSalle invariant principle, we have established sufficient conditions for the globally stability of each of the feasible equilibria of the system. As a result, we have shown the threshold for the permanence and extinction of the system. By Theorems 7-9, we see that (i) if $r r_{1}<d_{2}\left(r_{1}+d_{1}\right)$,

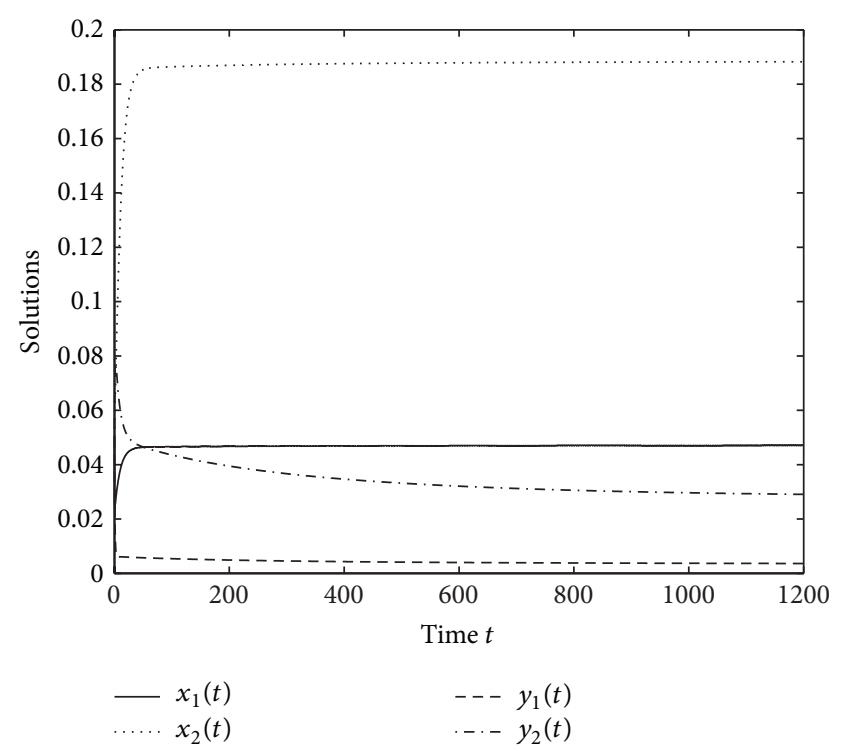

FIgURE 2: The temporal solution found by numerical integration of system (2) with $\tau=3$.

then both the prey and the predator population go to extinction; (ii) the prey species is permanent but the predator becomes extinct if and only if $0<\left(r r_{1}-d_{2}\left(r_{1}+d_{1}\right)\right) / a d_{2}<$ $d_{4}\left(r_{2}+d_{3}\right) /\left(a_{2} r_{2}-m d_{4}\left(r_{2}+d_{3}\right)\right)$; (iii) if $\underline{x}_{1}>\left(r r_{1}-d_{2}\left(r_{1}+\right.\right.$ $\left.\left.d_{1}\right)\right) / a d_{2}-d_{4}\left(r_{2}+d_{3}\right) /\left(a_{2} r_{2}-m d_{4}\left(r_{2}+d_{3}\right)\right)$ holds, then both the prey and predator species of system (2) are permanent.

\section{Conflict of Interests}

The authors declare that there is no conflict of interests regarding the publication of this paper.

\section{Acknowledgment}

This work was supported by the National Natural Science Foundation of China (no. 11101117).

\section{References}

[1] Y. Kuang and J. W.-H. So, "Analysis of a delayed two-stage population model with space-limited recruitment," SIAM Journal on Applied Mathematics, vol. 55, no. 6, pp. 1675-1696, 1995.

[2] W. Wang, "Global dynamics of a population model with stage structure for predator," in Advanced Topics in Biomathematics, L. Chen, S. Ruan, and J. Zhu, Eds., pp. 253-257, Word Scientific Publishing, 1997.

[3] W. Wang and L. Chen, "A predator-prey system with stagestructure for predator," Computers \& Mathematics with Applications, vol. 33, no. 8, pp. 83-91, 1997.

[4] Y. N. Xiao and L. S. Chen, "Global stability of a predator-prey system with stage structure for the predator," Acta Mathematica Sinica, vol. 20, no. 1, pp. 63-70, 2004.

[5] R. Xu, "Global stability and Hopf bifurcation of a predatorprey model with stage structure and delayed predator response," Nonlinear Dynamics, vol. 67, no. 2, pp. 1683-1693, 2012. 
[6] R. Xu, "Global dynamics of a predator-prey model with time delay and stage structure for the prey," Nonlinear Analysis. Real World Applications, vol. 12, no. 4, pp. 2151-2162, 2011.

[7] C. S. Holling, "The functional response of predators to prey density and its role in minicry and population regulation," Memoirs of the Entomological Society of Canada, vol. 45, pp. 360, 1965.

[8] Y. Kuang, Delay Differential Equation with Application in Population Synamics, vol. 191 of Mathematics in Science and Engineering, Academic Press, New York, NY, USA, 1993.

[9] B. D. Hassard, N. D. Kazarinoff, and Y. H. Wan, Theory and Applications of Hopf Bifurcation, vol. 41 of London Mathematical Society Lecture Note Series, Cambridge University Press, Cambridge, UK, 1981.

[10] J. Hale, Theory of Functional Differential Equation, Springer, Heidelberg, Germany, 1977.

[11] J. K. Hale and P. Waltman, "Persistence in infinite-dimensional systems," SIAM Journal on Mathematical Analysis, vol. 20, no. 2, pp. 388-395, 1989. 


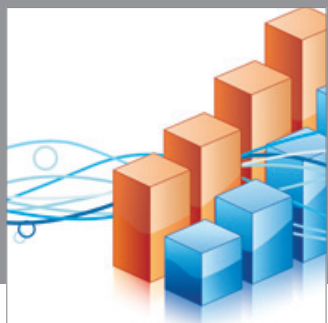

Advances in

Operations Research

mansans

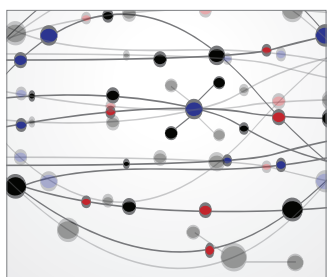

The Scientific World Journal
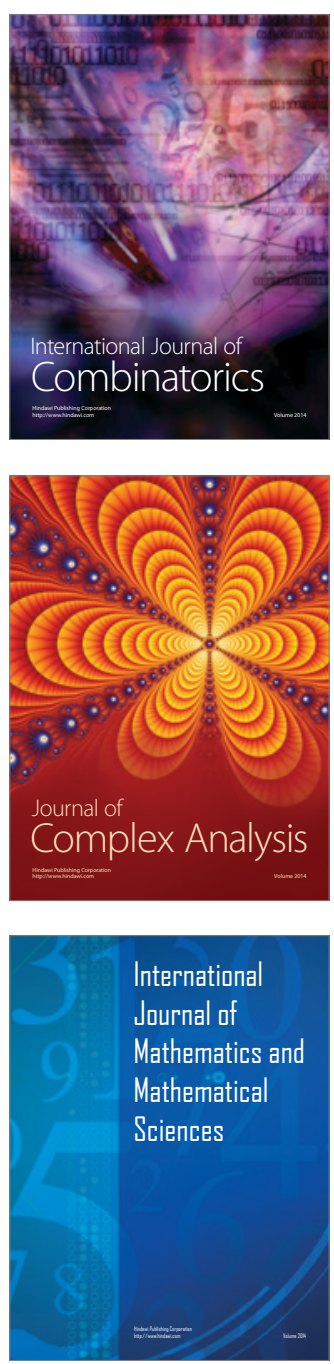
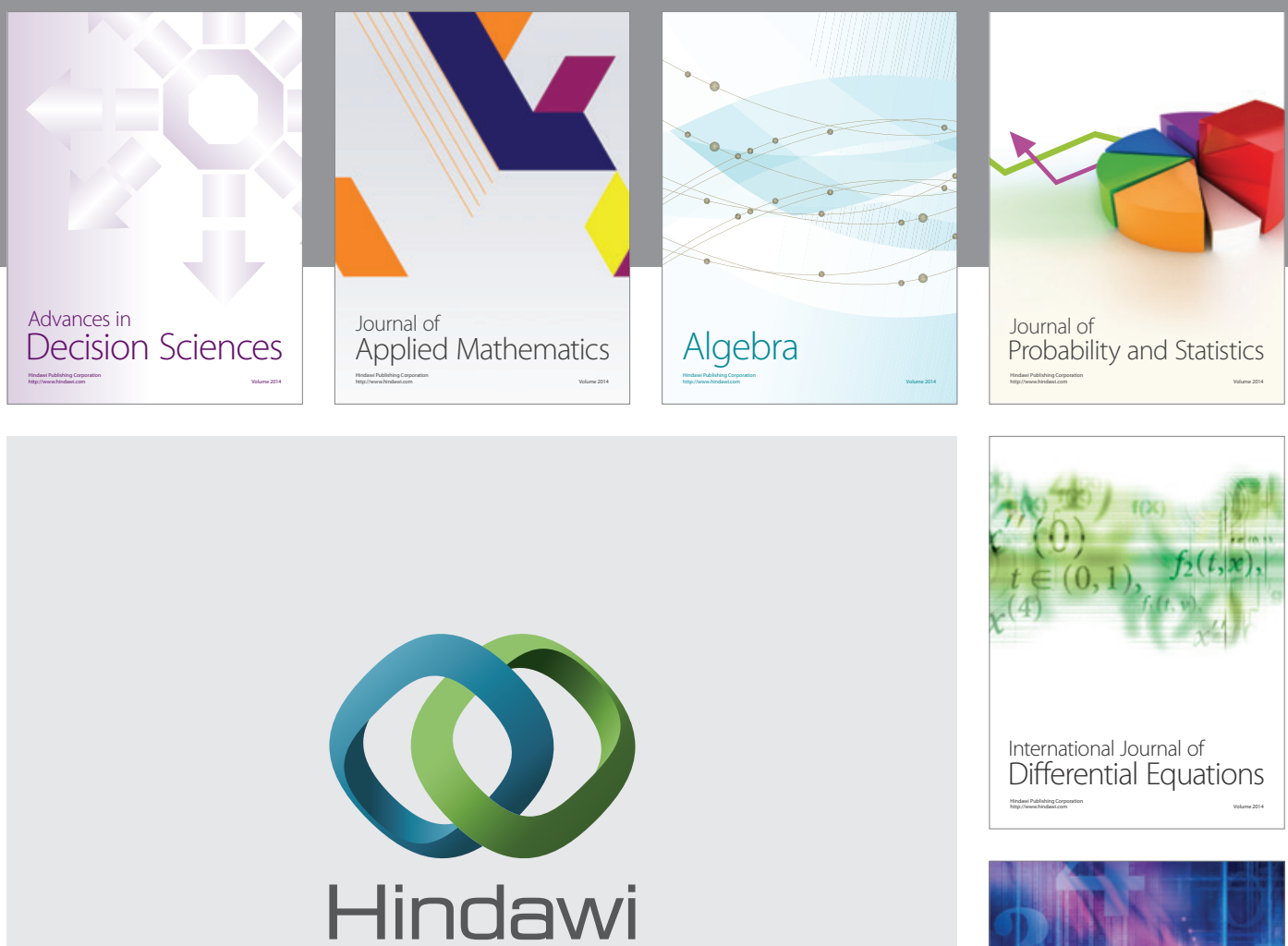

Submit your manuscripts at http://www.hindawi.com
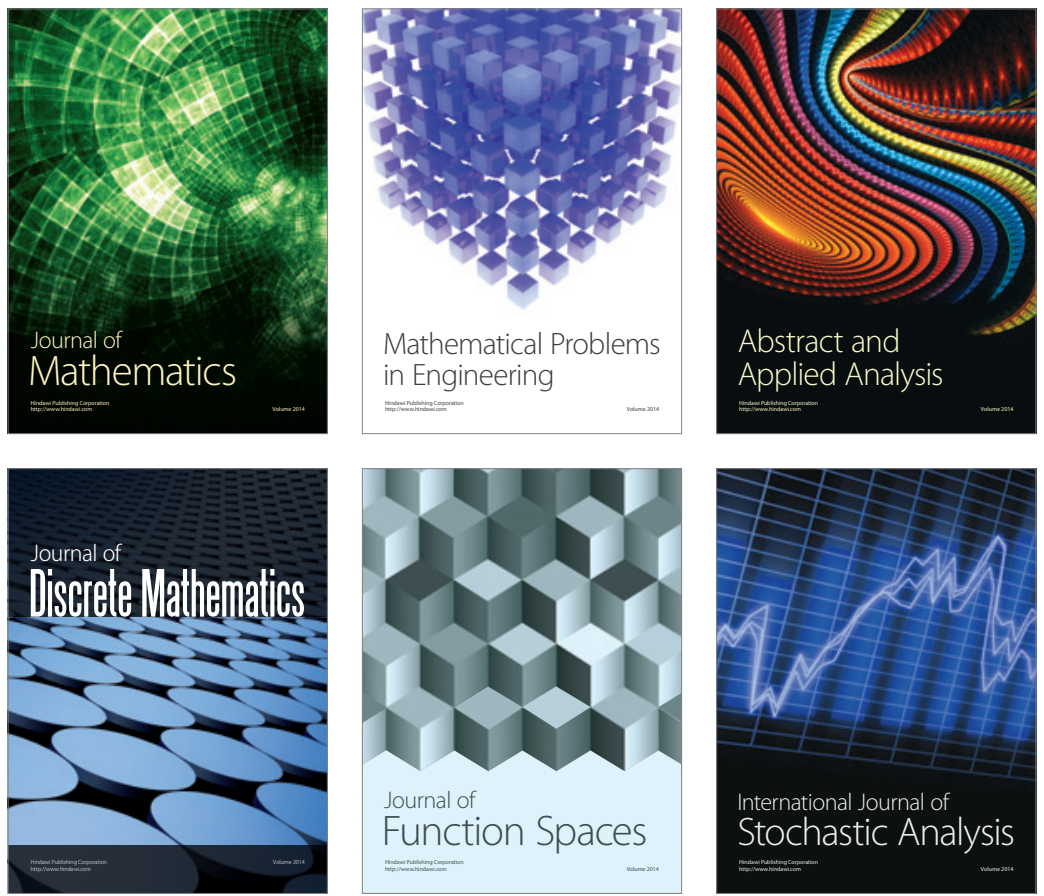

Journal of

Function Spaces

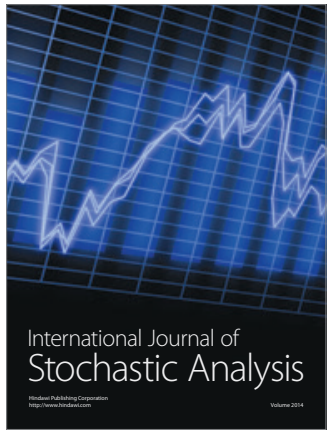

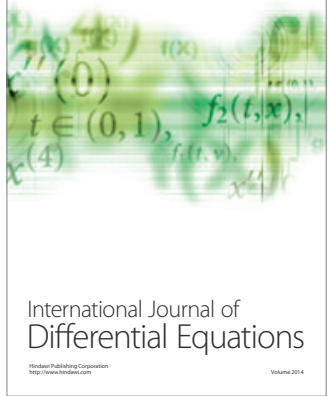
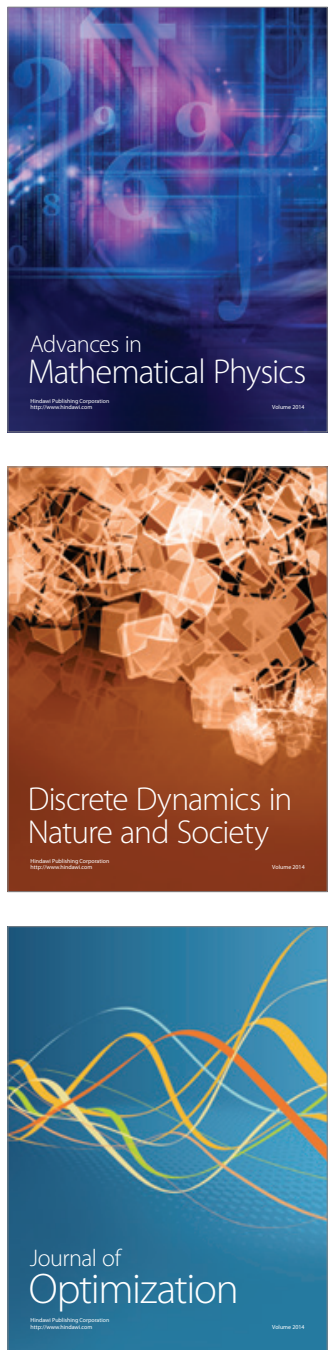